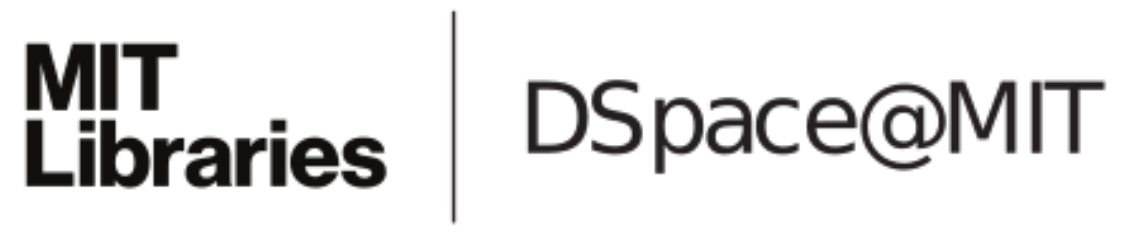

\author{
MIT Open Access Articles
}

Long-external-cavity distributed Bragg reflector laser with subkilohertz intrinsic linewidth

The MIT Faculty has made this article openly available. Please share how this access benefits you. Your story matters.

Citation: Lin, Qian et al. “Long-external-cavity Distributed Bragg Reflector Laser with Subkilohertz Intrinsic Linewidth.” Optics Letters 37.11 (2012): 1989. (C) 2012 Optical Society of America

As Published: http://dx.doi.org/10.1364/OL.37.001989

Publisher: Optical Society of America

Persistent URL: http://hdl.handle.net/1721.1/76646

Version: Final published version: final published article, as it appeared in a journal, conference proceedings, or other formally published context

Terms of Use: Article is made available in accordance with the publisher's policy and may be subject to US copyright law. Please refer to the publisher's site for terms of use. 


\title{
Long-external-cavity distributed Bragg reflector laser with subkilohertz intrinsic linewidth
}

\author{
Qian Lin, ${ }^{1}$ Mackenzie A. Van Camp, ${ }^{1}$ Hao Zhang, ${ }^{1}$ Branislav Jelenković, ${ }^{2}$ and Vladan Vuletić ${ }^{1, *}$ \\ ${ }^{1}$ Department of Physics, MIT-Harvard Center for Ultracold Atoms and Research Laboratory of Electronics, Massachusetts \\ Institute of Technology, Cambridge, Massachusetts 02139, USA \\ ${ }^{2}$ Institute of Physics, University of Belgrade, Serbia \\ *Corresponding author: vuletic@mit.edu
}

Received February 9, 2012; revised March 23, 2012; accepted March 24, 2012; posted March 27, 2012 (Doc. ID 162676); published May 30, 2012

\begin{abstract}
We report on a simple, compact, and robust $780 \mathrm{~nm}$ distributed Bragg reflector laser with subkilohertz intrinsic linewidth. An external cavity with optical path length of $3.6 \mathrm{~m}$, implemented with an optical fiber, reduces the laser frequency noise by several orders of magnitude. At frequencies above $100 \mathrm{kHz}$ the frequency noise spectral density is reduced by over $33 \mathrm{~dB}$, resulting in an intrinsic Lorentzian linewidth of $300 \mathrm{~Hz}$. The remaining lowfrequency noise is easily removed by stabilization to an external reference cavity. We further characterize the influence of feedback power and current variation on the intrinsic linewidth. The system is suitable for experiments requiring a tunable laser with narrow linewidth and low high-frequency noise, such as coherent optical communication, optical clocks, and cavity QED experiments. @ 2012 Optical Society of America
\end{abstract}

OCIS codes: $140.3425,140.2020,140.4780$.

Highly stabilized and ultranarrow-linewidth lasers are important for applications in atomic physics, quantum measurement, and quantum information science. The traditional extended-cavity diode lasers (ECDLs) [ 1$]$ and distributed Bragg reflector (DBR) lasers have typical linewidths of a few hundred kilohertz to a few ten megahertz, and specially fabricated DBR lasers have achieved an intrinsic linewidth of a few kilohertz [2,, 3$]$. Optical feedback from a resonator [4] is a common approach to reduce the linewidth of an ECDL, and recent systems [5-7] have demonstrated linewidth narrowing down to $7 \mathrm{kHz}$. Other linewidth narrowing schemes, such as active electronic stabilization to a high-finesse, ultrastable vertical cavity $[\underline{8}, \underline{9}]$, or an all-fiber Michelson interferometer [10], have achieved subhertz linewidth. However, due to finite loop gain at high frequencies, the high-frequency noise is usually not substantially reduced using those active stabilization methods. This may be detrimental in some applications, such as cavity sideband cooling [11,12], cavity spin squeezing [13], and other cavity QED applications [14], as well as coherent optical communication [15].

In this Letter, we present a tunable, long-externalcavity DBR laser with a 3000-fold reduction of its intrinsic linewidth to $300 \mathrm{~Hz}$. This is achieved by implementing a $3.6 \mathrm{~m}$ long external cavity using an optical fiber. The system exhibits noise suppression of over $33 \mathrm{~dB}$ at frequencies of $100 \mathrm{kHz}$ and above. Low-frequency noise due to mechanical or thermal fluctuations in the feedback path can be controlled by stabilizing the laser to a reference cavity using an active servo loop. Feedback-induced mode instability [16] is avoided by operating within a $13 \mathrm{~dB}$ wide range of feedback power.

We use a 780 nm DBR laser (Photodigm PH780DBR120T8-S) with $120 \mathrm{~mW}$ maximum output power as our source. The laser diode has a front facet reflectivity close to $1 \%$, effective gain region length of $1.8 \mathrm{~mm}$, and DBR reflectivity of $60 \%$ (all values are nominal values provided by manufacturer). The diode is temperature stabilized to slightly below room temperature. Throughout our measurements, we operated the laser at $100 \mathrm{~mA}(16 \mathrm{~mW}$ total output power). A beam splitter deflects $10 \%$ of the laser power into a $2 \mathrm{~m}$ long polarization-maintaining optical fiber that constitutes the feedback path (Fig. 1). An aperture is added before the angled fiber to block unwanted backreflection from the fiber tip. A mirror mounted on a piezoelectric transducer (PZT) reflects the light back into the fiber, and a quarterwave plate in combination with a polarizing beam splitter is used to adjust the feedback power. A maximum $-30 \mathrm{~dB}$ fractional power can be reflected back into the laser. (Henceforth we denote the fractional feedback power incident on the laser collimator as $p$, which may be less than the fractional power that is mode matched into the active region of the laser diode.) The externalcavity laser is set up on a $12^{\prime \prime} \times 18^{\prime \prime}$ aluminum baseplate and enclosed by a plastic case to reduce environmental perturbations.

In general, the effect of external cavity optical feedback depends on the feedback power, cavity length, and the phase of the feedback light, as summarized by Tkach

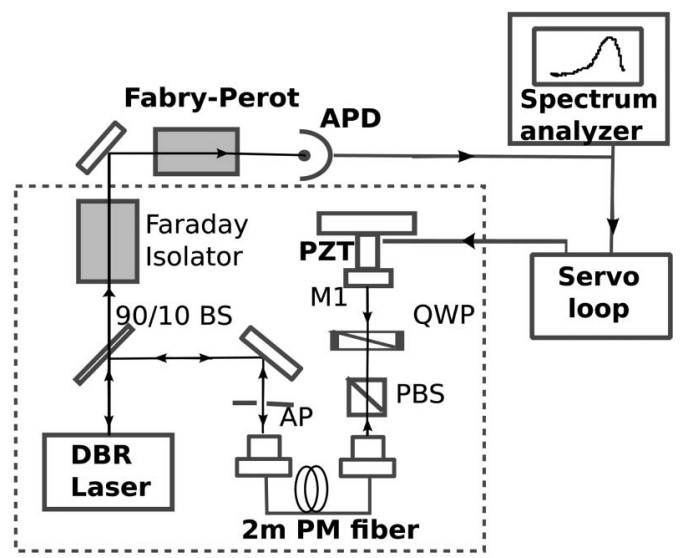

Fig. 1. Schematic setup for the long-external-cavity laser and the frequency noise characterization. M, mirror; AP, aperture; PM fiber, polarization-maintaining fiber; QWP, quarter-wave plate; BS, beam splitter; PBS, polarization beam splitter; $\mathrm{APD}$, avalanche photodiode; PZT, piezoelectric transducer. 
and Chraplyvy [16]. Stable, narrow-linewidth operation of a DBR laser with optical feedback is only possible over a certain range of feedback power. At a fixed cavity length, three regimes of operation are observed as the fractional feedback power $p$ is varied from $-65 \mathrm{~dB}$ to $-30 \mathrm{~dB}$. The feedback phase is adjusted by moving the mirror M1 (Fig. 1) with the PZT.

- Significant line broadening is observed for $p<-64 \mathrm{~dB}$. For $-64 \mathrm{~dB}<p<-49 \mathrm{~dB}$ and at certain feedback phases, the laser jumps at a rate over $1 \mathrm{kHz}$ between two adjacent external cavity modes separated by $42 \mathrm{MHz}$.

- For $-49 \mathrm{~dB}<p<-40 \mathrm{~dB}$, stable single-mode operation is maintained for all feedback phases. $-40 \mathrm{~dB}$ to $-36 \mathrm{~dB}$ defines a transition between two regimes where multimode operation occurs occasionally but quickly converges to a single mode. Weak sidebands at adjacent external cavity modes with over $25 \mathrm{~dB}$ suppression from the carrier were observed.

- For $-35 \mathrm{~dB}<p<-31 \mathrm{~dB}$, intermittent multimode operation starts to appear as the feedback phase is varied, and bi-stable operation with the laser jumping slowly $(<1 \mathrm{~Hz})$ between nonadjacent external cavity modes separated by as much as $0.5 \mathrm{GHz}$ is also observed. Nevertheless, it is still possible to maintain single-mode operation once the laser is frequency locked to some other reference, since the feedback phase is then stabilized. For $p>-31 \mathrm{~dB}$, multimode operation becomes dominant, and single-mode operation can only be achieved by increasing $p$ from a lower level carefully while controlling the feedback phase.

To measure the laser's frequency noise spectrum, we locked the laser to the half-transmission of a Fabry-Perot cavity with a free spectral range of $1.5 \mathrm{GHz}$ and linewidth of 4.0(2) MHz. The cavity transmission slope is used to convert power noise measured with a fast photodiode into frequency noise density $S_{\nu}(f)$. Stable locking achieved with a $15 \mathrm{kHz}$ bandwidth feedback loop to the externalcavity PZT maintains a constant frequency-to-power conversion ratio. Since the lock bandwidth is low, the noise spectrum above a few ten kilohertz directly reflects the effect of optical feedback. At sufficiently high noise frequencies, $S_{\nu}(f)$ becomes independent of frequency with a level of $S_{0}$, corresponding to a laser emission spectrum with Lorentzian wings whose equivalent intrinsic linewidth $\Delta \nu$ can be calculated using [17]

$$
\Delta \nu=\pi S_{0}^{2}
$$

We measured the noise spectrum from $10 \mathrm{kHz}$ to $1 \mathrm{MHz}$ for the long-cavity laser as well as the DBR laser without optical feedback. As shown in Fig. 2, the white noise $S_{0}$ starts at $300-400 \mathrm{kHz}$ for the bare DBR laser. For the long-cavity laser with cavity length $D=3.6 \mathrm{~m}$, suppression of over $30 \mathrm{~dB}$ due to optical feedback was observed for both the Lorentzian and the non-Lorentzian parts of the spectrum. A minimum intrinsic linewidth $\Delta \nu=0.29(5) \mathrm{kHz}$ is achieved at $p=-31 \mathrm{~dB}$, exhibiting a 3000-fold reduction in intrinsic linewidth compared to the bare laser. To verify the expected inverse quadratic dependence of $\Delta \nu$ on the external cavity length [툐], we

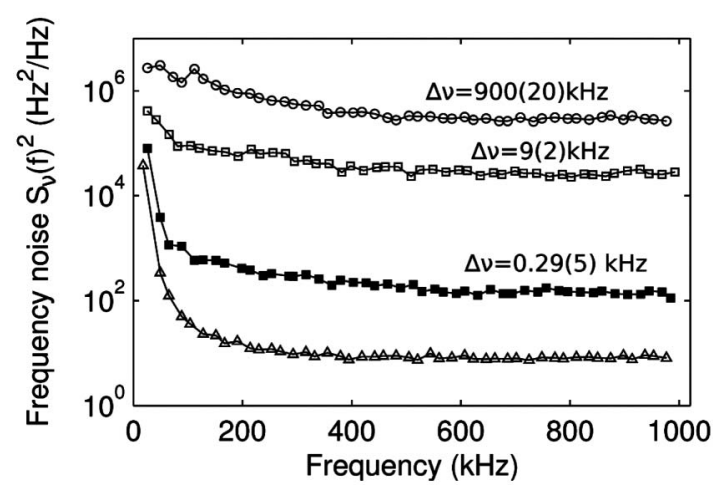

Fig. 2. Laser frequency noise spectral density for different setups. From top to bottom: bare DBR laser, short-external-cavity laser, long-external-cavity laser, and measurement noise floor. The feedback power is $-31 \mathrm{~dB}$ for both external cavities. The white-noise level $S_{0}$ at high frequency determines the intrinsic linewidth $\Delta \nu$ according to Eq. (1).

also set up a short-external-cavity laser using the same laser with cavity length $d=16.5(5) \mathrm{cm}$ and operated it at the same laser current and feedback power as the longexternal-cavity laser. The linewidth ratio for the two cavity lasers of $\Delta \nu_{d} / \Delta \nu_{D}=25(1) \mathrm{dB}$ is very close to the predicted value $[\underline{18}](D / d)^{2}=26.8(1) \mathrm{dB}$.

The dependence of the intrinsic linewidth $\Delta \nu$ on the fractional feedback power $p$ at fixed current, and on a variation in current at fixed feedback power is shown in Fig. 3. In general, a higher $p$ produces better noise suppression but makes the laser more susceptible to operating multimode. We found that for $p=-36 \mathrm{~dB}$ the noise density is only $3 \mathrm{~dB}$ higher than the minimum value (Fig. 3), but the laser remains stable, and frequency stabilization to an external reference can be easily achieved. At fixed $p$ and external cavity length $D$, the current can be tuned over a limited range $(\approx 250 \mu \mathrm{A})$ while the laser remains on the same mode. For $p=-39 \mathrm{~dB}$, it is observed that when decreasing the current, $\Delta \nu$ will increase gradually until the laser jumps to an adjacent external cavity mode. On the contrary, when increasing the laser current, a flat response in $\Delta \nu$ is followed by a sudden change into multimode oscillation. Thus, as long as one locks the laser close to the center of the flat response region, a $\pm 50 \mu \mathrm{A}$ current change can be tolerated without significant changes in $\Delta \nu$.

To tune the laser to a target frequency, one first changes the current at fixed cavity length, corresponding to the laser jumping over adjacent external cavity modes, until the mode closest to the target frequency is reached. Then the cavity length is tuned to change the laser
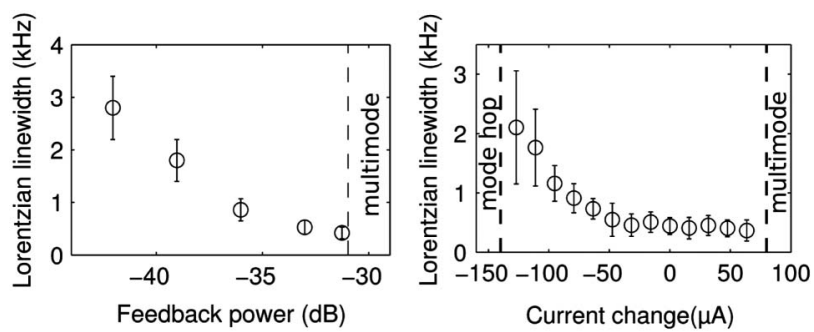

Fig. 3. The dependence of intrinsic laser linewidth $\Delta \nu$ on fractional feedback power $p$ (left) and on laser current near the operation point at $p=-39 \mathrm{~dB}$ and $I_{0}=100 \mathrm{~mA}$ (right). 
frequency continuously to the exact target frequency. Finally, the laser current is fine adjusted to obtain optimal linewidth narrowing. Mode-hop free tuning over $700 \mathrm{MHz}$ (more than 16 external cavity modes) can also be achieved by scanning the PZT and laser current simultaneously at an appropriate ratio. This tuning range was limited by the maximum stroke of our PZT. The optimal narrowing condition can generally be maintained during the scan, but may be limited by the nonlinearity of the PZT response.

Optical feedback reduces the high-frequency noise, but servo feedback is necessary to suppress the lowfrequency noise that is dominated by thermal and acoustic noise in the fiber. The free running laser exhibits a frequency stability of $\approx 600 \mathrm{kHz}$ over $20 \mathrm{~ms}$ and $\approx 3 \mathrm{MHz}$ over $1 \mathrm{~s}$ as measured relative to a reference laser locked to an ${ }^{87} \mathrm{Rb}$ atomic transition. To demonstrate frequency stabilization at the subkilohertz level, we locked the laser to a cavity with finesse $\mathcal{F}=2.3(2) \times 10^{4}$ (linewidth $\delta \nu_{c}=$ $120(10) \mathrm{kHz}$ ) with $5 \mu \mathrm{W}$ incident power and $p=-36 \mathrm{~dB}$ using the Pound-Drever-Hall technique [19]. The feedback loop includes a low-bandwidth feedback path to the PZT and an ac coupled, high-bandwidth (2 MHz) path to the laser current. The sideband frequency is chosen at $59.2 \mathrm{MHz}$ to prevent the up-converted modulation signal from triggering adjacent external cavity modes at integer multiples of $42 \mathrm{MHz}$. An rms frequency error of 0.65 (5) $\mathrm{kHz}$ relative to the reference cavity was calculated from the error signal in a $50 \mathrm{kHz}$ bandwidth.

In conclusion, we have built a subkilohertz, tunable DBR laser in a compact long-external-cavity setup by fiber-coupling the feedback light. A $35 \mathrm{~dB}$ reduction in the high-frequency noise was demonstrated. Different regimes of operation under varied levels of feedback power were characterized and are in agreement with previous results [16]. We have investigated the linewidth and mode stability as a function of feedback power and laser current variation. Owing to its low cost, ease of alignment and robust performance, we believe the system can be used for a wide range of high-precision and ultra-lownoise experiments.

This work was supported in part by the National Science Foundation (NSF), the Defense Advanced Research Projects Agency (DARPA) (QuASAR), and the
United States Army Research Office (ARO). Q. Lin acknowledges support from the Undergraduate Research Opportunity Program at MIT, M. A. Van Camp from the NSF IGERT program, and B. Jelenkovic from the Ministry of Education and Science of Serbia, under grant 45016.

\section{References}

1. L. Ricci, M. Weidemuller, T. Esslinger, A. Hemmerich, C. Zimmermann, V. Vuletic, W. Konig, and T. W. Hänsch, Opt. Commun. 117, 541 (1995).

2. S. Spießberger, M. Schiemangk, A. Wicht, H. Wenzel, G. Erbert, and G. Tränkle, Appl. Phys. B 104, 813 (2011).

3. S. Spießberger, M. Schiemangk, A. Sahm, A. Wicht, H. Wenzel, A. Peters, G. Erbert, and G. Tränkle, Opt. Express 19, 7077 (2011).

4. B. Dahmani, L. Hollberg, and R. Drullinger, Opt. Lett. 12, 876 (1987).

5. J. Labaziewicz, P. Richerme, K. R. Brown, I. L. Chuang, and K. Hayasaka, Opt. Lett. 32, 572 (2007).

6. Y. Zhao, Y. Peng, T. Yang, Y. Li, Q. Wang, F. Meng, J. Cao, Z. Fang, T. Li, and E. Zang, Opt. Lett. 36, 34 (2011).

7. K. Hayasaka, Opt. Lett. 36, 2188 (2011).

8. A. D. Ludlow, X. Huang, M. Notcutt, T. Zanon-Willette, S. M. Foreman, M. M. Boyd, S. Blatt, and J. Ye, Opt. Lett. 32, 641 (2007).

9. J. Alnis, A. Matveev, N. Kolachevsky, T. Udem, and T. W. Hänsch, Phys. Rev. A 77, 053809 (2008).

10. F. Kefelian, H. Jiang, P. Lemonde, and G. Santarelli, Opt. Lett. 34, 914 (2009).

11. K. W. Murch, K. L. Moore, S. Gupta, and D. M. Stamper-Kurn, Nat. Phys. 4, 561 (2008).

12. M. H. Schleier-Smith, I. D. Leroux, H. Zhang, M. A. V. Camp, and V. Vuletic, Phys. Rev. Lett. 107, 143005 (2011).

13. M. H. Schleier-Smith, I. D. Leroux, and V. Vuletic, Phys. Rev. Lett. 104, 073604 (2010).

14. P. R. Berman, ed., Cavity Quantum Electrodynamics (Advances in Atomic, Molecular, and Optical Physics Supplement 2) (Academic, 1994).

15. K. Kikuchi, Electron. Lett. 23, 885 (1987).

16. R. W. Tkach and A. R. Chraplyvy, J. Lightwave Technol. 4, 1655 (1986).

17. D. S. Elliott, R. Roy, and S. J. Smith, Phys. Rev. A 26, 12 (1982).

18. C. H. Henry, J. Lightwave Technol. 4, 298 (1986).

19. R. W. P. Drever, J. L. Hall, F. V. Kowalski, J. Hough, G. M. Ford, A. J. Munley, and H. Ward, Appl. Phys. B 31, 97 (1983). 